\title{
SCADA Based Automated Bottle Quality \& Quantity Detection and Filling System by Using PLC
}

\author{
Mr.P.Choudesh Varma ${ }^{1}$ and Mr.G.Venkateswarlu ${ }^{2}$ \\ ${ }^{1}$ CVR College of Engineering, Department of EIE., Hyderabad, India \\ Email: choudesh82@gmail.com \\ ${ }^{2}$ CVR College of Engineering, Department of EIE., Hyderabad, India \\ Email: venkigummadilli@gmail.com
}

\begin{abstract}
In this paper, we propose an automated bottle quality, quantity detection and filling system in a manufacturing department using a PLC (Programmable Logic Controller) and SCADA. SCADA stands for Supervisory Control and Data Acquisition system. The main objective of the project has four processing stages. Firstly, detect the fault of the bottle by using optical sensor. In the second stage, remove the faulted bottle from the conveyer belt using electronic pusher. In the next stage, detect the quantity (size) of the bottle using proximity sensor. In the final stage, bottle filling process regarding size of the bottle. The quality and quantities of the physical system in the field is sensed by the sensors and fed as inputs to the PLC which in turn sent to PC through RS-232 for necessary processing. A PLC program is developed based on the signals received from the PLC input module, process the signals and send the output signals to the PLC output modules, which is programmed to actuate the necessary electronic actuator.

The SCADA screens are developed regarding four processing stages on PC using Wonderware-Intouch 9.0 SCADA software. The Allen Bradley micrologix 1000 PLC to be link up with the supporting SCADA Wonderware-Intouch 9.0 by giving necessary tag names. With the help of SCADA, simulation of the physical system is also developed to monitor the quantities to be measured and status of the equipment in the plant from field point.
\end{abstract}

Index Terms: PLC, SCADA, I/O Modules Actuator, Proximity and optical sensors.

\section{INTRODUCTION}

Modern bottle quality, quantity detection and filling process is truly sophisticated, this can even be done automatically with computer controlled equipment. The methods of process variables controlling have been changed rapidly in recent years. Bottle quality, quantity detection and filling systems are an important Application in the fields of pharmacy, food processing, fertilizer industries. The main aim of the project has four processing stages.

1. Detect the fault of the bottle using optical sensor

2. Remove the faulted bottle from the conveyer belt using electronic pusher.

3. Detect the quantity (size) of the bottle using proximity sensors.

4. Bottle filling process regarding size of the bottle.

Fault detection: Optical sensor is placed one side of the conveyor belt and it detects the cracks present on the bottle (If the bottle has a crack then transmitted light is reflected and then optical sensor detects [7]the reflected light) if any. Then PLC sends control signals to the pusher through PLC O/P modules and then faulted bottle is removed by the pusher from the conveyor belt.

Quantity detection and filling: Proximity sensors are placed side of the conveyor belt to detect the size of the bottle. Regarding size of the bottle, signals are transmitted from proximity sensors (P.S-2 \& P.S-3) to PLC through PLC I/O modules [1] then the data will be updated in the PLC programming. A another capacitive proximity switch is placed one side of the conveyor belt and it detects the bottle which comes near to the filling tank and then the running of conveyor belt stops and solenoid valve is opened, then bottle is filled for a few seconds mentioned in the timer based on quantity of the bottle which is taken in the PLC program.

A capacitive proximity switch which is used for the bottle filling purpose is also used for the counting purpose. The capacitive proximity switch output is given to the counter. Whenever the switch output is $\mathrm{ON}$ then there will be a increment in the counter. The counter can be seen in the SCADA screen [4]. By this, we can know how many bottles are present on the conveyor belt. Every updated status of field devices can be monitored on the SCADA screen.

The paper is organized as follows. Section I Introduction to bottle quality, quantity detection, filling and techniques used for Measurement of physical variables considered and controlling in our process.

Section II: Block diagram of the application considered 
Section III: Field devices and specifications

Section IV: Programmable logic controllers

Section V: Programming language \& results

Section VI: SCADA \&, results

Section VII: Conclusition and future enhancement

Section VIII: Acknowledgement

Section IX: references.

\section{II -BLOCK DIAGRAM OF THE APPLICATION}

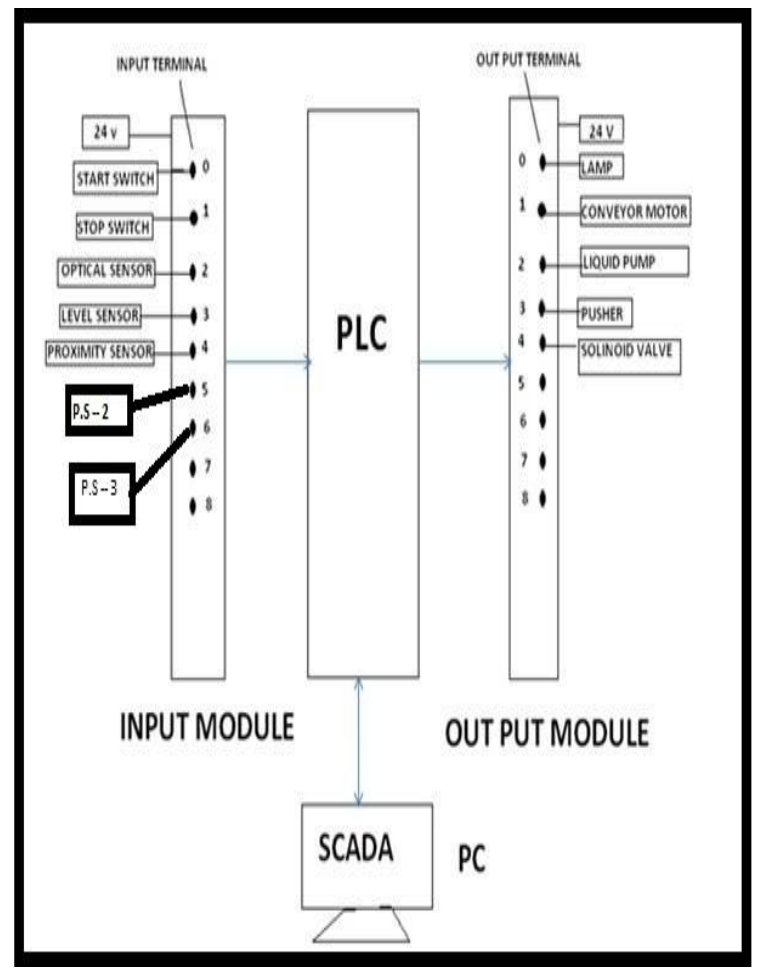

Figure 1.Block diagram

This block diagram explains the inputs, outputs and their interfacing with the PLC and SCADA. In this all inputs and outputs are connected to the input module and output module of PLC respectively. The PLC is interfaced with SCADA using RS-232 by using respective drivers. The PLC requires the power supply of $24 \mathrm{~V}$; it is given by using SMPS (switched-mode power supply).

Here, the inputs are proximity switches, level sensors; master start and master stop switches. Three capacitive proximity switches namely proximity sensor, proximity sensor (P.S-2) and proximity sensor (P.S-3) is connected to the PLC as inputs and also an optical switch namely fault detecting sensor is connected to the PLC as input. Level sensors and master start switch and master stop switch are also connected to the PLC as inputs. A pusher, solenoid valve, two motors namely pump motor and conveyor motor are connected as outputs to the PLC as shown in the fig 1 .

The process behind in this block diagram is, whenever the ON button in SCADA is turned ON then the conveyor belt motor will be ON then the conveyor belt tends to run. When there is a crack on the bottles which are placed on conveyor belt is detected then the optical sensor will be ON, then a signal is send to the PLC and the PLC sends a signal to the pusher which is an output device to ON, then the pusher pushes the fault bottle out from the conveyor belt.

Whenever the bottle comes near to P.S-2 (half Ltr bottle) and P.S-3(1Ltr bottle) sensors then which sends signals (quantity of the bottle) to the PLC through I/O modules. Whenever the bottle comes under the solenoid valve the proximity switch will ON then sends the signal to the PLC and the PLC sends signal to the conveyor belt motor through output modules to OFF and solenoid valve to open for few minutes or seconds regarding size of the bottle, Which we mentioned in the timer [1] [2] the process going in the field and status of field devices can be monitored on SCADA screen..

The proximity switch is also connected to the counter to count the bottles running on the conveyor belt. Here we use two level sensors arranged in the overhead tank at bottom and top. When water level in the overhead tank is decreased from the bottom level sensor then the pump motor will ON. When the water level in the overhead tank reaches the top level sensor then the motor will be OFF. Here the ON and OFF operation of pump motor is done by the signal sent by the PLC.

\section{FIELD DEVICES AND SPECIFICATIONS}

The devices which are interfaced with PLC I/O modules [1] are called as field devices like sensors and actuators. The following devices are used to implement the automated bottle quality, quantity detection and filling system.

Frame: In industries series of wheels are placed under the thick and strong conveyor belt which has the capability to bear the weight of the bottles .In industries there is heavy machinery to fill the bottles and capping it .In our project, a frame is designed with iron and wood materials. The length of the frame is 5.5 feet and the breadth of the frame is 1 feet. In our project, the whole apparatus which we used is placed on the frame and it has to bear all the weight of the apparatus.

On the frame, bearings are placed on either side and get welded. In industries there are series of wheels which are used to give support to the conveyor belt but here we are not using such type of heavy machinery, so we designed a flat surface sheet made up of wood material and it gives support to the conveyor belt to weigh the bottles when they are going to fill. Here a motor is also welded on one side of the frame and it is connected to the toothed wheel which is attached with bearings, so motor is used to run the conveyor belt, the frame which is made up of iron and wood material is shown below Fig.2. 


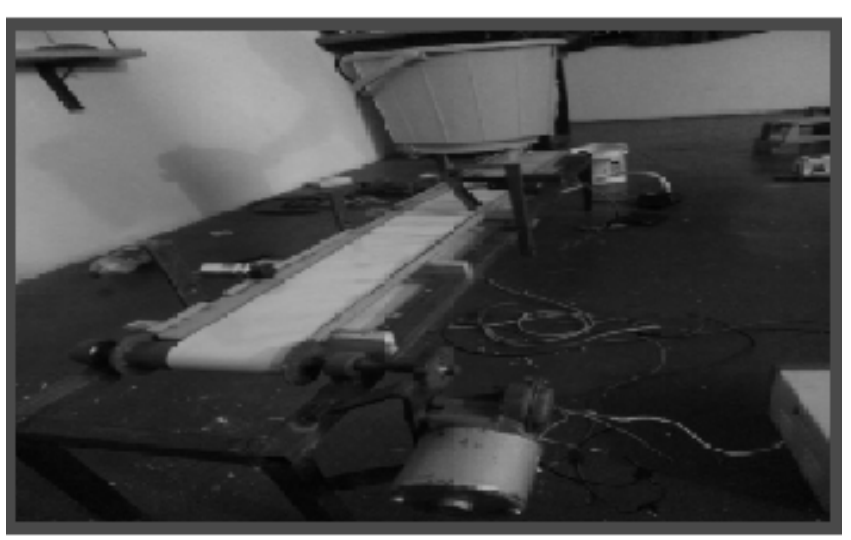

Figure.2 Frame with conveyor belt

Electrical pusher: In industries various pushers are used to push the fault bottles are any fault material present on the conveyor belt. Some of the pushers are mechanical pushers, hydraulic pushers, electronic pushers etc.In our application, we used electronic pusher to push the fault bottle on the conveyor belt found if any. Which is operating for $24 \mathrm{v}$ input voltage. We preferred electronic pusher is shown in Fig.3 because it is less cost than other pushers and it is suitable for our application. Pusher is connected to PLC output module terminal-3.we have to consider the terminal at the time of writing the PLC programming, give the address to that particular terminal as

$\mathrm{O}: 0 / 3$ where $\mathrm{O}$ represents output module and3 represents pusher is connected to $3^{\text {rd }}$ terminal of output module

The working of the pusher [8] used in this project is, inside the pusher there is a coil and adjacent to the coil there is a magnetic piece. Whenever current passes through the coil then it acts as magnet. The magnetic piece and the current carrying coil will have the same poles, so like poles repel each other. So the magnetic piece gets repelled. To this magnetic piece we connect a spring and hence it acts as the pusher.

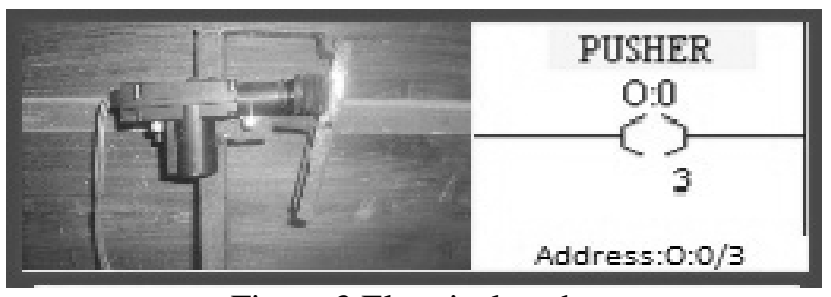

Figure.3 Electrical pusher

Level switch: The main application of this level switch (Fig.4) is to sense the water level in large water tanks and sumps. Level switch is connected to PLC input module terminal-3.we have to consider the terminal at the time of writing the PLC programming[1] [2] , give the address to that particular terminal as I: $0 / 3$ where I represents output module and 3 represents level switch is connected to $3^{\text {rd }}$ terminal of input module.
The rating of this level switch is $10 \mathrm{~A}$ resistive at $230 \mathrm{~V}$ AC. The switch type present in this switch is SPDT. The switching angle is $+/-45 \mathrm{deg}$. The medium temperature is max 50deg and medium pressure is max $0.1 \mathrm{pa}$ (1bar). The medium density is $\mathrm{min} 0.8 \mathrm{gm} /$ cubic $\mathrm{cm}$. Housing material is polypropylene. Cable material is PVC or neoprene. Cable diameter is $9 \mathrm{~mm} / 3 \times 1 \mathrm{sq}$ $\mathrm{mm}$. Cable length is 5 metres.

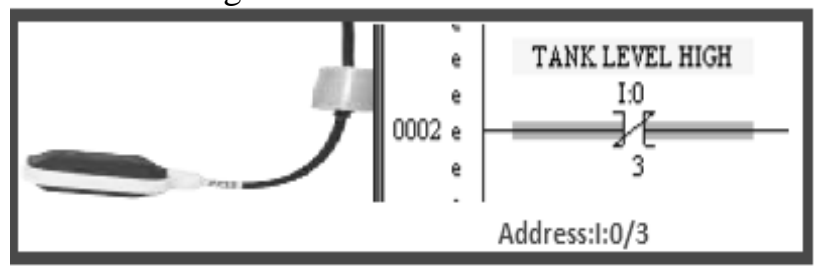

Figure.4 Level switch

DC motor: It is used to run the conveyor belt. This is connected to PLC O/P module through relay $24 \mathrm{v}$ operating voltage. Whenever the master start button is pressed then PLC sends the control signals to the O/P modules (terminal-1).then the motor is ON through the relay. .we have to consider the terminal at the time of writing the PLC programming, give the address to that particular terminal as $\mathrm{O}: 0 / 1$ where $\mathrm{O}$ represents output module and 1 represents motor is connected to $3^{\text {rd }}$ terminal of output module.

It is based on the principle that when a current carrying conductor is placed in a magnetic field it experiences a mechanical force. Now a day's DC motor (shown in Fig.5) place a vital role in most of the industrial areas.

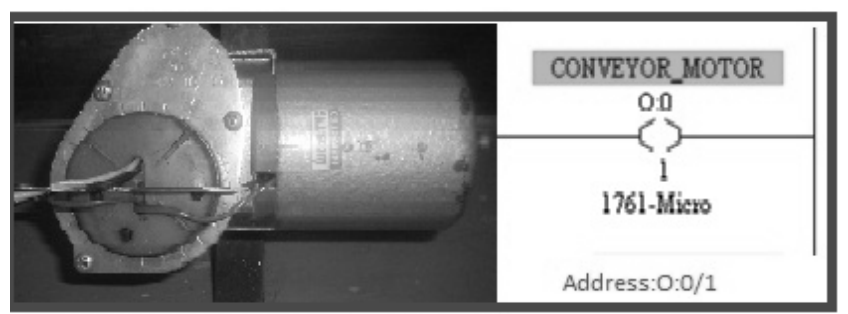

Figure.5 conveyor motor

Solenoid valve: This is connected to PLC O/P module (Terminal4).Whenever the bottle reaches near to filling tank then PLC sends the control signals to solenoid valve to fill the bottle. We have to consider the terminal at the time of writing the PLC programming; give the address to that particular terminal as $\mathrm{O}: 0 / 4$ where 4 represents level switch is connected to $3^{\text {rd }}$ terminal of input module

A Solenoid valve (Fig.6) is electromechanically operated valve, the valve is controlled by an electric current through a solenoid: in the case of a two-port valve the flow is switched on or off; in the case of a three-port valve, the outflow is switched between the two outlet ports. Multiple solenoid valves can be placed together on a manifold. 
If the valve is open when the solenoid is not energized, then the valve is termed normally open (N.O.). Similarly, if the valve is closed when the solenoid is not energized, then the valve is termed normally closed internally piloted valves are used in dishwashers and irrigation systems where the fluid is water, the pressure might be 80 pounds per square inch (550 kPa) and the orifice [9] diameter might be $3 / 4$ in $(19 \mathrm{~mm})$. A direct acting solenoid valve typically operates in 5 to 10 milliseconds. The operation time of a piloted valve depends on its size; typical values are 15 to 150 milliseconds.

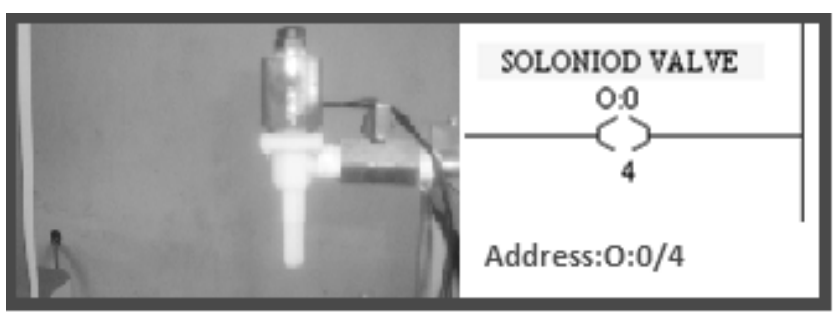

.Figure.6 solenoid valve

Proximity switch: A capacitive switch (Fig.7) is a proximity switch that detects nearby objects (bottles) by their effect on the electrical field created by the sensor. Here we are using three proximity sensors, one sensor is for to detect the bottle nearby filling tank that is connected to PLC input module $4^{\text {th }}$ terminal addressed as I:0/4 and two more sensors are proximity sensor (P.S2) and proximity sensor (P.S-3) are connected to the PLC input modules to detect the quantity of the bottle, P.S-2 (half Ltr bottle) addressed as I:0/5 and P.S-3(1Ltr bottle) addressed as I:0/6.

Simple capacitive switches [9] have been commercially available for many years, and have found a niche in nonmetallic object detection, but are limited to short ranges, typically less than $1 \mathrm{~cm}$.

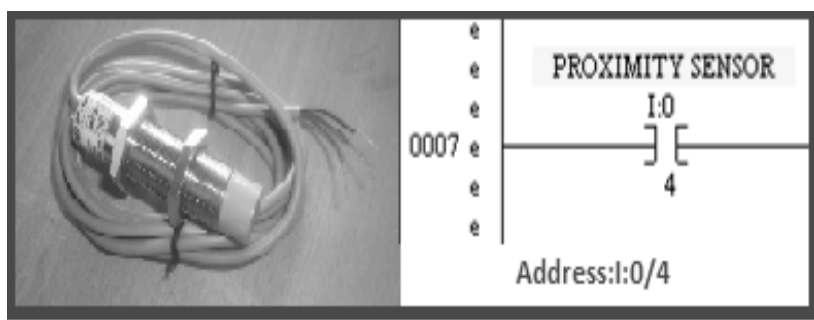

Figure.7 proximity switch

\section{PROGRAMMABLE LOGIC CONTROLLER}

Programmable logic controllers (fig.8) are nothing but a type of solid state devices designed to perform logic functions previously accomplished by electromechanical relays mainly used in industrial equipments. These industrial equipments may range from a small ON-OFF switch to a heavy machine. Any component in industry that needs a control can be controlled by using PLC. The main advantage of using PLC is that long and tedious programming can be eliminated. The programming for PLC is done by using the ladder logic diagram. The ladder logic consists of blocks which are in the form of ladders.

In our project PLC (Allen Bradley micrologix 1000 PLC) [1] is used to monitor and provide the required indication and control like Detect the fault of the bottle , Remove the faulted bottle, Detect the quantity (size) of the bottle, filling process and also. The liquid level is controlled using the PLC. We have two levels namely, low and high levels. By using the level sensor, the PLC makes the motor ON and the tank is filled. As soon as the water reaches the high level the motor is stopped.

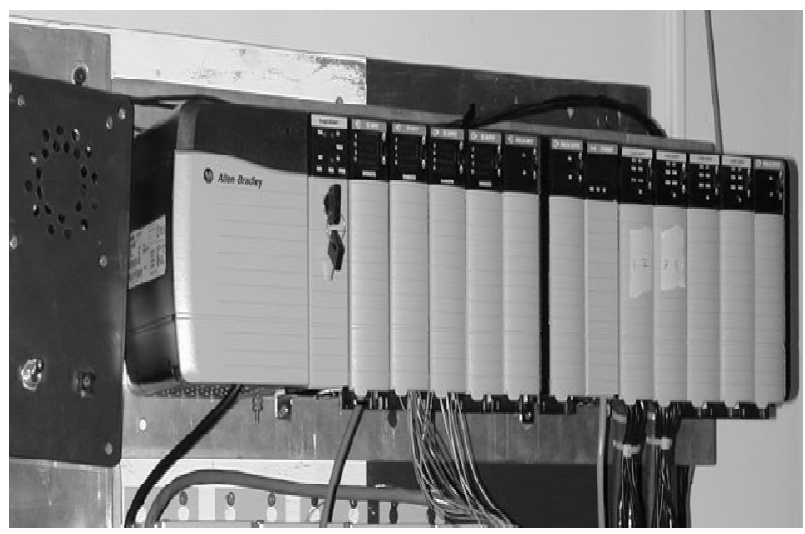

Figure.8 Allen Bradley micrologix 1200 PLC

I/O Modules: The input module has terminals to which outside process electric signals, generated by sensors or transducers are entered. The output module has terminals to which output signals are sent to activate relays, solenoids, various solid-state switching devices, motors and displays. An electronic system for connecting I/O modules [1] [2] to remove locations can be added if needed. The actual operating process and PLC control can be thousands of feet from the CPU and its I/O modules

The input module (Fig.9) performs four tasks electronically

1. First it senses the presence or absence of an input signal at each of its input terminals..The input signal tells what switch, sensor or other signal is $\mathrm{ON}$ or OFF in the process being controlled. 


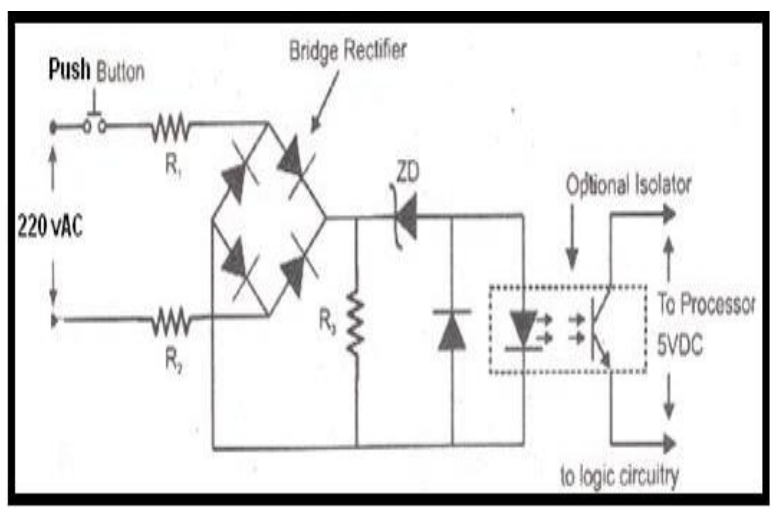

Figure.9.Input module

2. Second, it converts the input signal for high, or on, to a DC level usable by the module's electronic circuit. For a low or OFF input signal, no signal is converted, indicating OFF.

3. Third, the input module carries out electronic isolation by electronically isolating the input module from its input.

4. Finally, its electronic circuit must produce an output, via output logic, to be sensed by the PLC CPU.

The output module (Fig.10) operates in the opposite manner from the input module.

A DC signal from the CPU is converted through each module section to a usable output voltage; either AC or DC.A signal from the CPU is received by the output module logic, one for each scan.

If the CPU signal code matches the assigned number of the module, the module section is turned ON. If the matching CPU signal is received, it goes through an isolation stage. Isolation is necessary so that any erratic voltage surge from the output device does not get back into the CPU and cause damage.

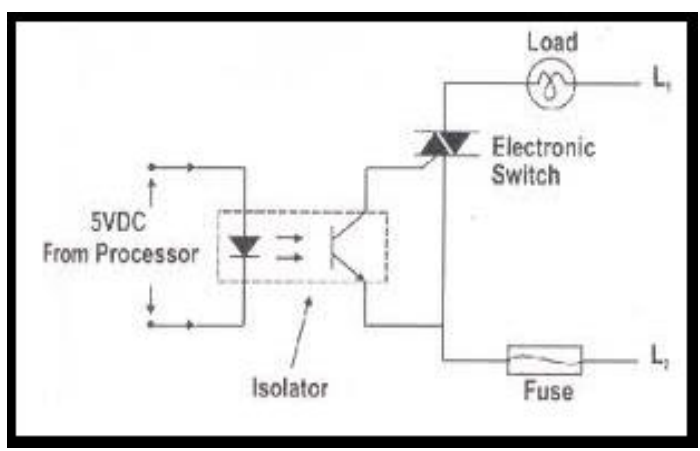

Figure.10.output modules
The isolator output is then transmitted to switching circuitry or an output relay. The output of a module section may be through a relay, or a DC or AC output.

\section{PROGRAMMING LANGUAGE}

PLC programs are typically written in a special application on a personal computer, and then downloaded by a direct-connection cable or over a network to the PLC. We have to Write the programme by considering the connections of field devices to the PLC input and output modules. The programme should satisfy the processing steps of our application. The program is stored in the PLC either in battery-backed-up RAM [1] or some other non-volatile flash memory. Often, a single PLC can be programmed to replace thousands of relays.

Under the IEC 61131-3 standard, PLCs can be programmed using standards-based programming languages, in this we are using ladder programming language

LADDER DIAGRAM (LD): Ladder logic is a programming Language (Fig.11) that represents a program by a graphical diagram based on the circuit diagrams of relay-based logic hardware. It is primarily used to develop software for Programmable Logic Controller (PLCs) used in industrial control applications. The name is based on the observation that programs in this language resemble ladders, with two vertical rails and a series of horizontal rungs between them [1] [3].

The following instructions are the desired PLC programming instructions to write the programme.

1. Normally open

2. Normally closed

3. Timer

4. Counter

Addressing format

Input->I: 0/0, I: 0/2, I: 0/0, I: 1/0, I: $2 / 3$

Output->O: 0/0, O: 0/1, O: 1/0, O: 2/4 

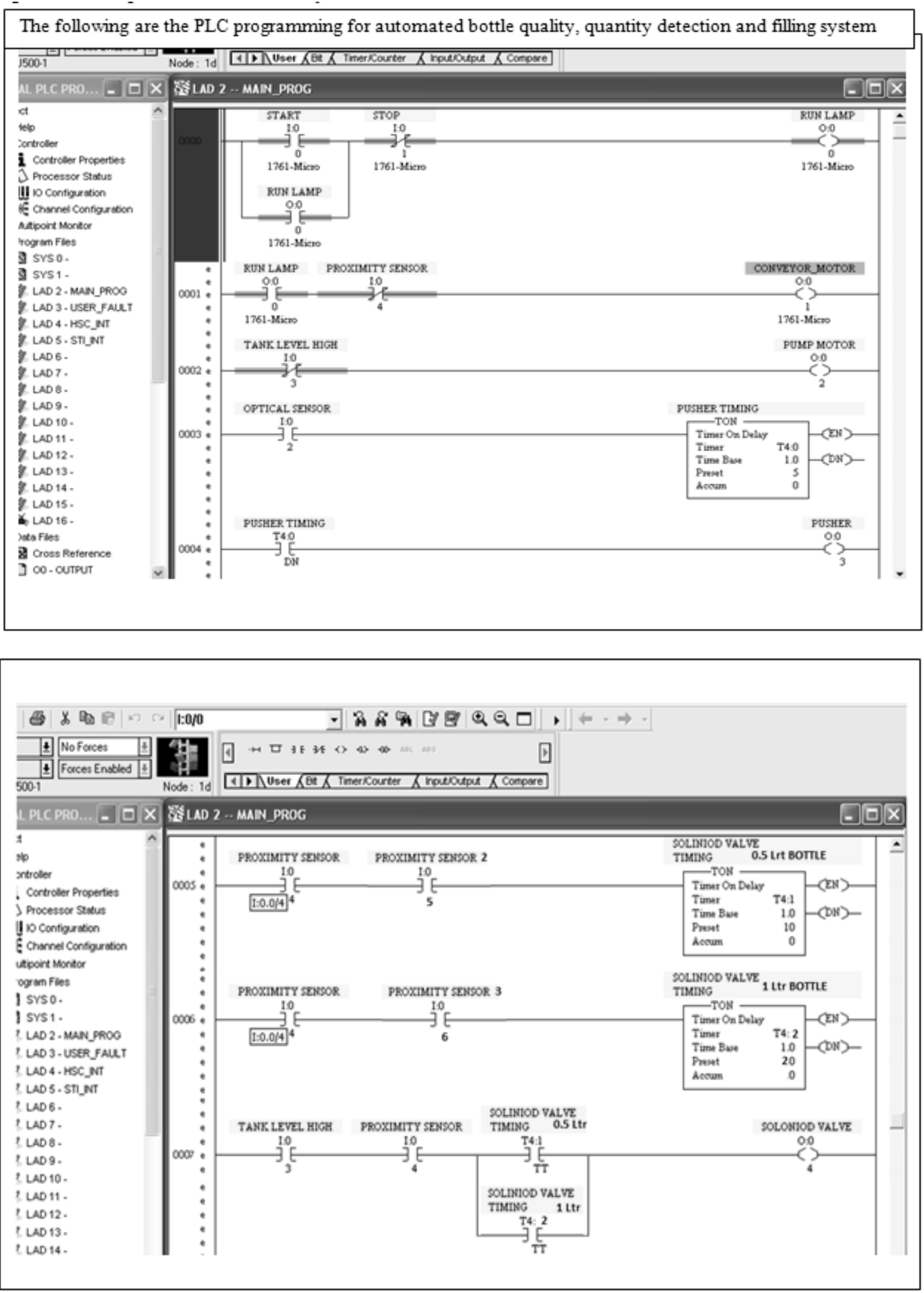


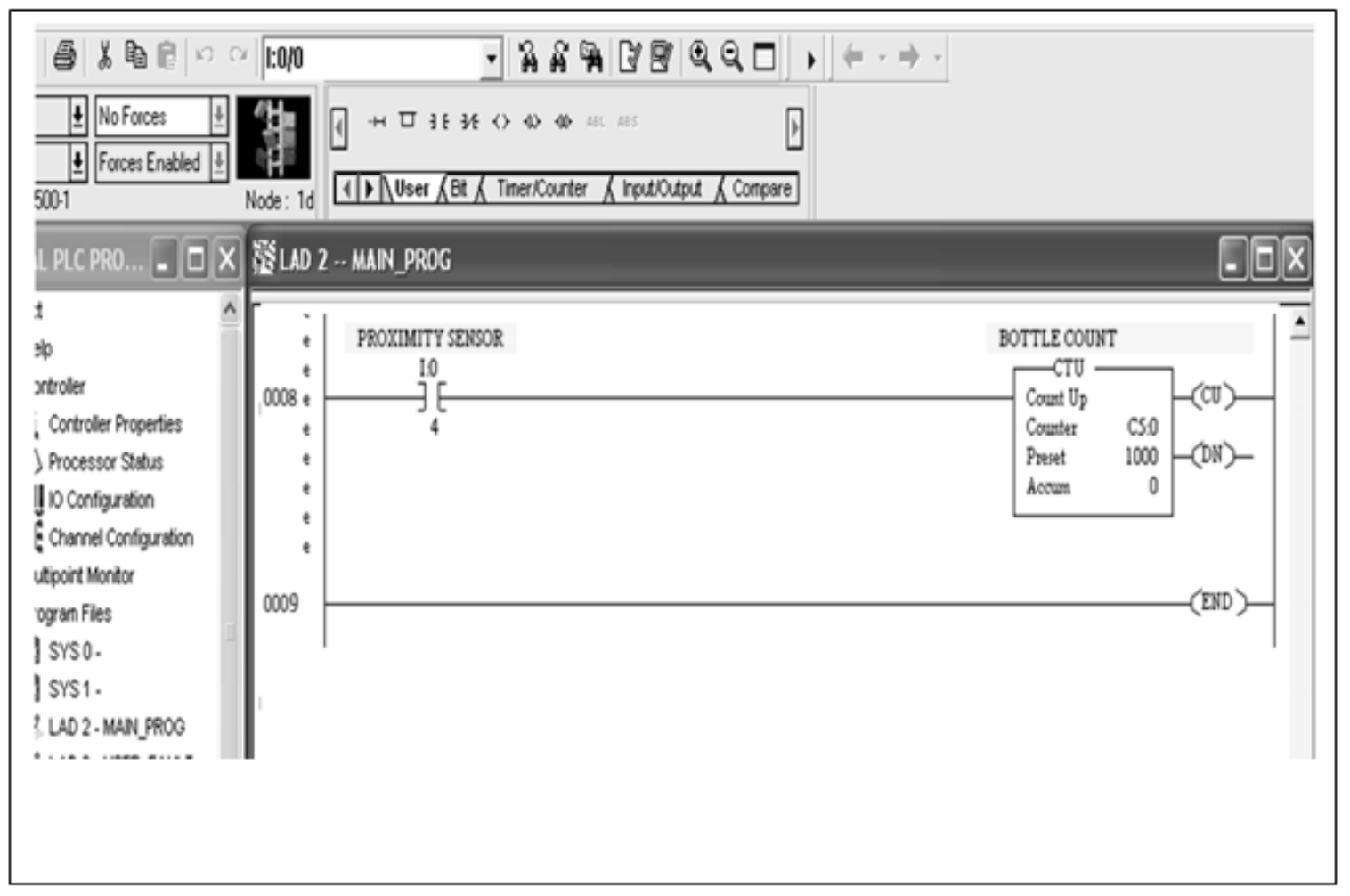

Figure.11.PLC programming

\section{SCADA}

In this project we are using Wonder ware InTouch SCADA. For 25 years Wonder ware InTouch has been the world's number one human machine interface (HMI).SCADA screens were developed based on the field devices which are interfaced to the PLC input and output modules. We can monitor and control the status of every field device from the SCADA system.

SCADA (supervisory control and data acquisition) [4] is a type of Industrial control system (ICS). Industrial control systems is computer controlled systems that monitor and control industrial processes that exist in the physical world. SCADA systems historically distinguish themselves from other ICS systems by being large scale processes that can include multiple sites, and large distances. Industrial processes include those of manufacturing, production, power generation, fabrication and refining, and may run in continuous, batch, repetitive, or discrete modes.

Whatever the symbols dragged into the screen from the graphics symbolic factory that could be link up with the PLC programming by considering the Tag Name(Fig.12) and instruction address then only we can monitoring and control the status of field devices.

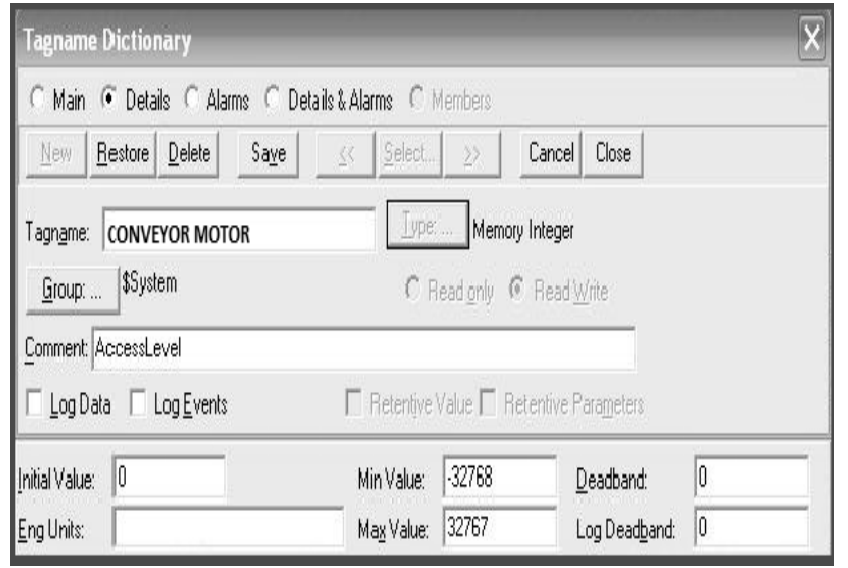

Figure.12.Tag Name Dictionary

Common system components: A SCADA system usually consists of the following subsystems:

1. A human machine interface or HMI (fig.13) is the apparatus or device which presents process data to a human operator [5] [2], and through this, the human operator monitors and controls the process.

2. SCADA is used as a safety tool as in lock-out-tagout. A supervisory (computer) system, gathering (acquiring) data on the process and sending commands (control) to the process.

3.Remote Terminal Units (RTUs) connecting to sensors in the process, converting sensor signal to digital data 
and sending digital data to the supervisory system. Programmable Logic Controller (PLCs) used as field devices because they are more economical, versatile, flexible, and configurable than special-purpose RTUs.

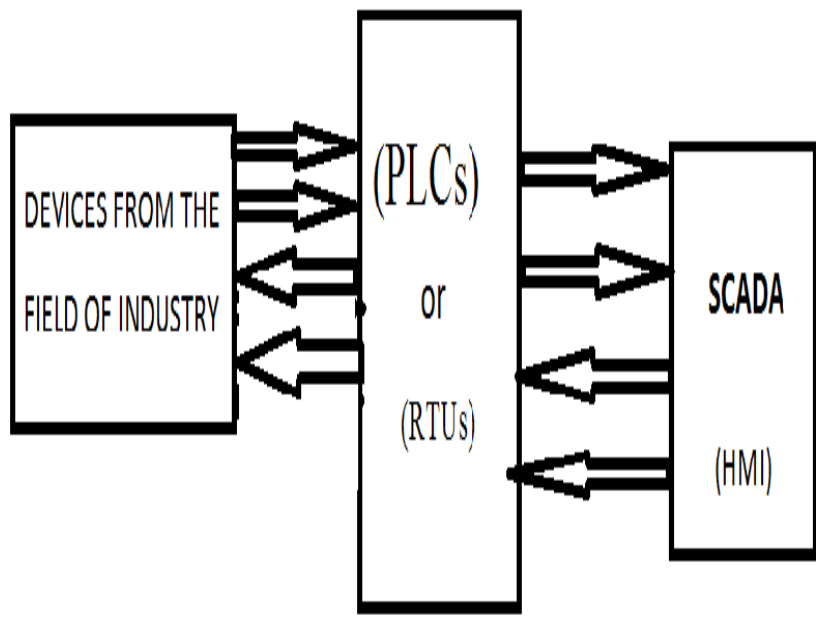

Figure.13.Human machine interface with field of industry

Wonder ware InTouch SCADA : The primary offering, Wonder ware InTouch SCADA, employs a development canvas with a drag-and-drop paradigm and a symbol library(Fig.14), which allows users to drag a graphic symbol from the library and drop it onto the canvas. From there, the symbols can be connected in a way that reflects the design of a plant floor.
Applications designed in Wonder ware InTouch SCADA enable plant engineers and operators to visualize plant floor operations and to issue operational commands such as START and STOP to one or more Programmable Logic Controllers either remotely or locally.

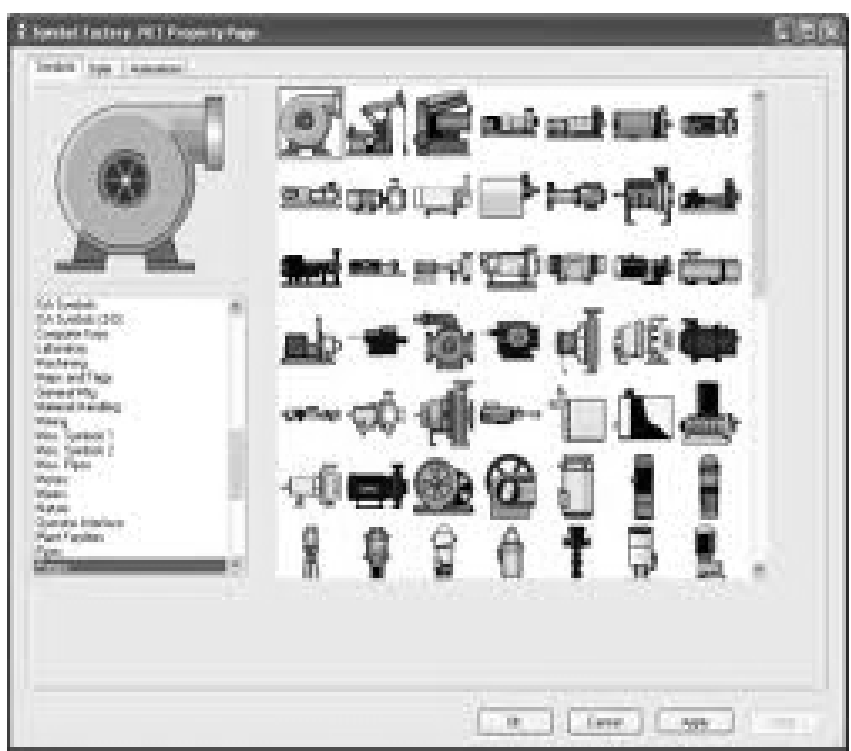

Figure.14.symbol factory

The result is shown in Fig.15. SCADA screen for automated bottle quality, quantity detection and filling system

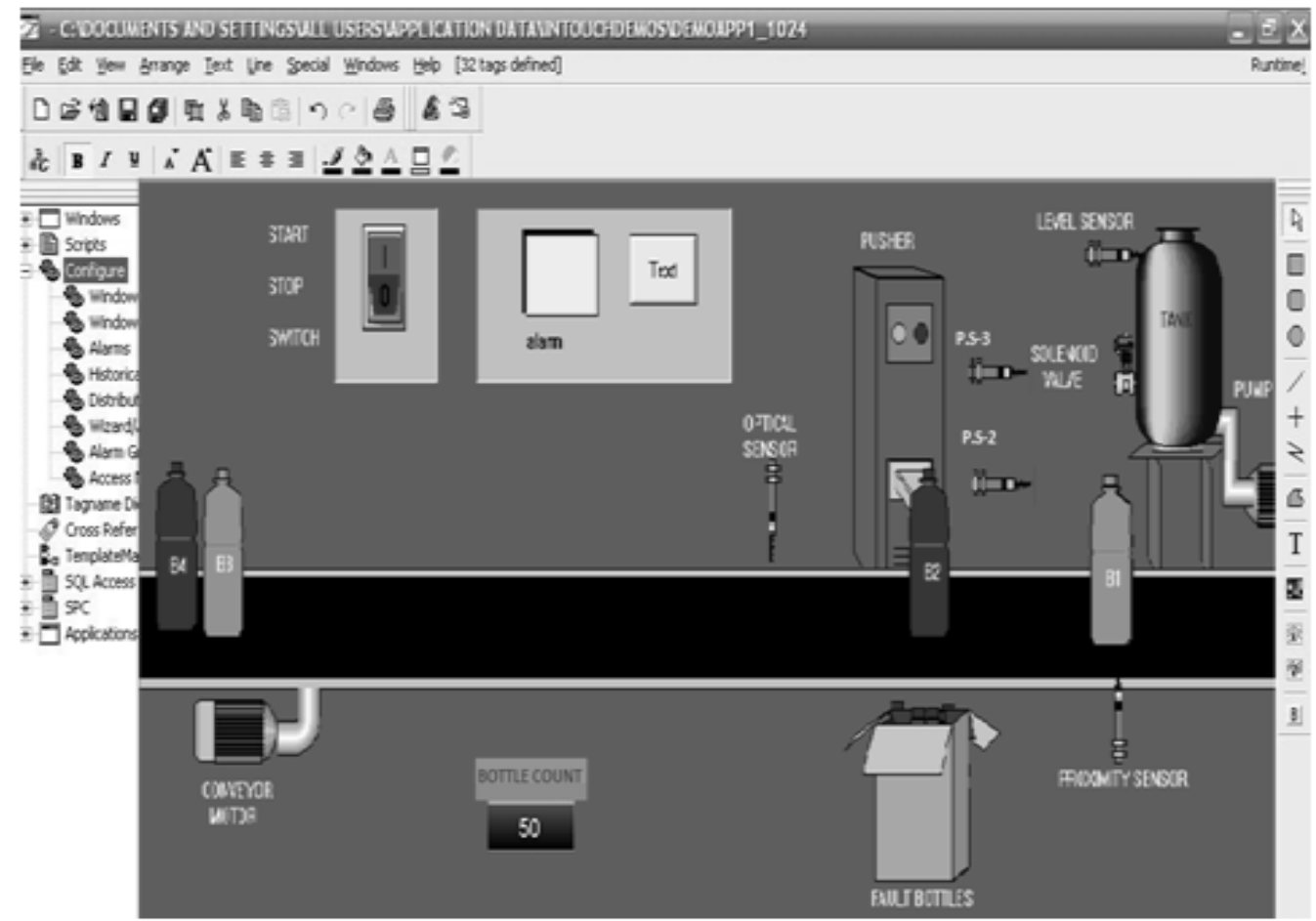

Figure.15. SCADA screen for automated bottle quality, quantity detection and filling system 


\section{CONCLUSION AND FUTURE ENHANCEMENT}

Automated bottle quality, quantity detection and filling system using PLC and SCADA has been successfully constructed and designed. The system that is produced can be modified to be better if some of the electrical devices and system are upgraded and improved.

The theory and concept of the automatic quality, quantity detection and filling water system is based on the PLC and SCADA. In electrical design, the features and functions of the electrical components are required to determine the system requirement.

Furthermore, the theoretical of the wiring system is required for connecting the inputs and outputs devices to PLC. In programming design, understandings of the desired control system and how to use the Ladder Diagram to translate the machine sequence of operation are the most important parts, because it has direct effect on the system performance. The main aim in this process is to apply PLC to design automatic filling water system and all objectives in this project were successfully done as planned.

Actually, a weakness from the project can be taken as future works so that the improved system will be better in terms of performance. So that, there are several recommendations or suggestions that we can take to increase performance in this project. The performance of automated bottle quality, quantity detection and filling system can be increased based on two recommendations which are;

The system that is proposed now is using only one sensor that is IR sensor to detect position of bottle. It will be better if we add more sensors in this system like a flow sensor to detect water flow or use level sensor to detect water level. Thus, the system will be more sensitive as there will be more sensing points. Besides using PLC as controller, the other controller can be used in the future work. However, many factors must be considered like cost, practically and others.

\section{REFERENCES}

[1]. John. W .Webb Ronald A Reis , Programmable Logic Controllers - Principles and Applications, Fourth edition, Prentice Hall Inc., New Jersey, 1998.

[2]. PC Interfacing and Data Acquisition Techniques for Measurement, Instrumentation and Control. By Kevin James; Elsevier.

[3]. Manual book, 'A Beginner's Guide to PLC', version 2.1. Singapore: Omron.2001.

[4]. SCADA: supervisory control and data acquisition by Stuart A.Boyer.

[5]. Securing SCADA system, in author "Ronald L.Krutz".

[6]. PLC Based Automated Packing Station Using National Instrument's Lab VIEW Software Package, International Conference on Computing and Control Engineering (ICCCE 2012).

[7]. Lasers and Optical Engineering - by Das P., Springer's International Students Edition, 1991.

[8]. A course in Electrical and Electronic Measurements and Instrumentation, A.K. Sawhney, Dhanpatrai \& Co.

[9] .Modern Electronic Instrumentation and Measurement techniques - by A.D. Helfrick and W. D. Cooper, Pearson/Prentice Hall of India. 